\title{
Semientwining Structures and Their Applications
}

\author{
Florin F. Nichita, ${ }^{1}$ Deepak Parashar, ${ }^{2,3}$ and Bartosz Zieliński ${ }^{4}$ \\ ${ }^{1}$ Institute of Mathematics "Simion Stoilow" of the Romanian Academy, P.O. Box 1-764, 014700 Bucharest, Romania \\ ${ }^{2}$ Cambridge Cancer Trials Centre, Department of Oncology, University of Cambridge, \\ Addenbrookes Hospital, (P.O. Box 279) Hills Road, Cambridge CB2 0QQ, UK \\ ${ }^{3}$ MRC Biostatistics Unit Hub in Trials Methodology Research, University Forvie Site, Robinson Way, Cambridge CB2 OSR, UK \\ ${ }^{4}$ Department of Theoretical Physics and Informatics, University of Łódź, Pomorska 149/153, 90-236 Łódź, Poland \\ Correspondence should be addressed to Deepak Parashar; dp409@cam.ac.uk
}

Received 6 October 2012; Accepted 31 October 2012

Academic Editors: W. de Graaf, V. Drensky, and S. Yang

Copyright (C) 2013 Florin F. Nichita et al. This is an open access article distributed under the Creative Commons Attribution License, which permits unrestricted use, distribution, and reproduction in any medium, provided the original work is properly cited.

Semientwining structures are proposed as concepts simpler than entwining structures, yet they are shown to have interesting applications in constructing intertwining operators and braided algebras, lifting functors, finding solutions for Yang-Baxter systems, and so forth. While for entwining structures one can associate corings, for semientwining structures one can associate comodule algebra structures where the algebra involved is a bialgebra satisfying certain properties.

\section{Introduction and Preliminaries}

Quantum groups appeared as symmetries of integrable systems in quantum and statistical mechanics in the works of Drinfeld and Jimbo. They led to intensive studies of Hopf algebras from a purely algebraic point of view and to the development of more general categories of Hopftype modules (see [1] for a recent review). These serve as representations of Hopf algebras and related structures, such as those described by the solutions to the Yang-Baxter equations.

Entwining structures were introduced in [2] as generalized symmetries of noncommutative principal bundles and provide a unifying framework for various Hopf-type modules. They are related to the so-called mixed distributive laws introduced in [3].

The Yang-Baxter systems emerged as spectral-parameter independent generalization of the quantum Yang-Baxter equation related to nonultra-local integrable systems $[4,5]$. Interesting links between the entwining structures and YangBaxter systems have been established in $[6,7]$. Both topics have been a focus of recent research (see, e.g., [8-13]).

In this paper, we propose the concepts of semientwining structures and cosemientwining structures within a generic framework incorporating results of other authors alongside ours. The semientwining structures are some kind of entwining structures between an algebra and a module which obey only one-half of their axioms, while cosemientwining structures are kind of entwining structures between a coalgebra and a module obeying the other half of their axioms. The main motivations for this terminology are the new constructions which require only the axioms selected by us (constructions of intertwining operators and YangBaxter systems of type II or liftings of functors), our new examples of semientwining structures, simplification of the work with certain structures (Tambara bialgebras, lifting of functors, braided algebras, and Yang-Baxter systems of type I), the connections of the category of semientwining structures with other categories, and so forth. Let us observe that while for entwining structures one can associate corings, for semientwining structures one can associate comodule algebra structures provided the algebra involved is a bialgebra with certain properties (see Theorem 9).

The current paper is organised as follows. Section 2 contains the newly introduced terminology with examples, new results, and comments. Section 3 is about some of the applications of these concepts, namely, new constructions of intertwining operators and braided algebras, lifting functors, 
and the presentations of Tambara bialgebras and of (new families of) Yang-Baxter systems (of types I and II).

The main results of our paper are Theorems 19, 22, 24, 40, and 41 . Theorems 29 and 31 are mentioned in the context of stating some of our results. Theorem 34 is used to prove Theorem 40, while Theorem 37 is related to Theorem 38 .

Unless otherwise stated, we work over a commutative ring $R$. Unadorned tensor products mean tensor products over $R$.

For any $R$-module $V, T(V)$ denotes tensor algebra of $V$. In section 3.5, we work over a field $\mathbb{K}$. For $V$ an $R$-module, we denote by $I: V \rightarrow V$ the identity map. For any $R$-modules $V$ and $W$ we denote by $\tau=\tau_{V, W}: V \otimes W \rightarrow W \otimes V$ the twist map, defined by $\tau_{V, W}(v \otimes w)=w \otimes v$. Let $\phi: V \otimes V \rightarrow$ $V \otimes V$ be an $R$-linear map. We use the following notations: $\phi_{12}=\phi \otimes I, \phi_{23}=I \otimes \phi, \phi_{13}=\left(I \otimes \tau_{V, V}\right)(\phi \otimes I)\left(I \otimes \tau_{V, V}\right)$.

Definition 1. An invertible $R$-linear map $\phi: V \otimes V \rightarrow V \otimes V$ is called a Yang-Baxter operator if it satisfies

$$
\phi_{12} \circ \phi_{23} \circ \phi_{12}=\phi_{23} \circ \phi_{12} \circ \phi_{23} \text {. }
$$

Remark 2. Equation (1) is usually called the braid equation. It is a well-known fact that the operator $\phi$ satisfies (1) if and only if $\phi \circ \tau_{V, V}$ satisfies the quantum Yang-Baxter equation (if and only if $\tau_{V, V} \circ \phi$ satisfies the quantum Yang-Baxter equation):

$$
\phi_{12} \circ \phi_{13} \circ \phi_{23}=\phi_{23} \circ \phi_{13} \circ \phi_{12} \text {. }
$$

\section{Semientwining Structures and Related Structures}

Definition 3 (Semientwining Structures). Let $A$ be an $R$ algebra, and let $B$ be an $R$-module, then the $R$-linear map $\psi: B \otimes A \rightarrow A \otimes B$ is called a (right) semientwining map if it satisfies the following conditions for all $a, a^{\prime} \in A$, $b \in B$ (where we use a Sweedler-like summation notation $\left.\psi(b \otimes a)=a_{\alpha} \otimes b^{\alpha}\right):$

$$
\begin{gathered}
\psi\left(b \otimes 1_{A}\right)=1_{A} \otimes b, \\
\psi\left(b \otimes a a^{\prime}\right)=a_{\alpha} a_{\beta}^{\prime} \otimes b^{\alpha \beta} .
\end{gathered}
$$

If $B$ is also an $R$-algebra, and a semientwining map satisfies additionally

$$
\begin{array}{r}
\psi\left(1_{B} \otimes a\right)=a \otimes 1_{B}, \quad \psi\left(b b^{\prime} \otimes a\right)=a_{\alpha \beta} \otimes b^{\beta} b^{\prime \alpha}, \\
\forall a \in A, \forall b, b^{\prime} \in B,
\end{array}
$$

then the semientwining map is called an algebra factorization (in the sense of [14]).

If $B$ is a coalgebra and satisfies

$$
\begin{gathered}
a_{\alpha} \varepsilon\left(b^{\alpha}\right)=a \varepsilon(b), \\
a_{\alpha} \otimes b_{(1)}^{\alpha} \otimes b_{(2)}^{\alpha}=a_{\alpha \beta} \otimes b_{(1)}^{\beta} \otimes b_{(2)}^{\alpha}, \quad \forall a \in A, \quad \forall b \in B,
\end{gathered}
$$

then $\psi$ is called a (left-left) entwining map [2].
Remark 4. Let $q \in R$. The following are examples of semientwining structures. Note that they do not have natural algebra factorization structures in general.

(1) Let $A$ be an $R$-algebra, then the $R$-linear map $\gamma_{q}: A \otimes$ $A \rightarrow A \otimes A, \gamma_{q}(b \otimes a)=1 \otimes b a+q b a \otimes 1-q b \otimes a$ is a semientwining map. Notice that $\gamma_{q}$ is a Yang-Baxter operator (according to [15]).

(2) Let $A$ be an $R$-algebra, then the $R$-linear map $\eta_{q}: A \otimes$ $A \rightarrow A \otimes A, \eta_{q}(b \otimes a)=q(b a-a b) \otimes 1+a \otimes b$ is a semientwining map. Notice that $\eta_{q}$ is a Yang-Baxter operator related to Lie algebras (see, e.g., [16]).

(3) Let $A$ be an $R$-algebra, and let $M$ be a right $A$-module. Then the $R$-linear map $\phi: M \otimes A \rightarrow A \otimes M, \phi(m \otimes$ a) $=1 \otimes m a$ is a semientwining map.

The proof of the next lemma is direct; the second statement is a well-known result.

Lemma 5. If $\psi: B \otimes A \rightarrow A \otimes B$ is a semientwining map, then

(i) $A \otimes B$ becomes a right $A$-module with the operation $(a \otimes b) * a^{\prime}=a a_{\alpha}^{\prime} \otimes b^{\alpha}$;

(ii) moreover, if $B$ is an algebra, we can define a bilinear operation

$$
\begin{aligned}
& \cdot(A \otimes B) \otimes(A \otimes B) \longrightarrow(A \otimes B), \\
& (a \otimes b) \otimes\left(a^{\prime} \otimes b^{\prime}\right) \longmapsto a a_{\alpha}^{\prime} \otimes b^{\alpha} b^{\prime},
\end{aligned}
$$

and $\cdot$ is an associative and unital multiplication on $A \otimes B$ if and only if $\psi$ is an algebra factorization.

Remark 6. Some authors call the above map $\psi$ a twisting map; see, for example, [17], where a unifying framework for various twisted algebras is provided.

Remark 7. Suppose that $A$ is a right $H$-comodule algebra (where $H$ is a bialgebra), and $B$ is a right $H$-module. Then

$$
\psi_{H}: B \otimes A \longrightarrow A \otimes B, \quad b \otimes a \longmapsto a_{(0)} \otimes b a_{(1)}
$$

is a semientwining map. Moreover, if $B$ is an $H$-module algebra, then $\psi_{H}$ thus defined is an algebra factorization. Finally, if $B$ is an $H$-module coalgebra, then $\psi$ is an entwining map, and $(A, H, B)$ is called a Doi-Koppinen structure (see [13]).

Remark 8. Let $A$ be an $R$-algebra. We define the category of semientwining structures over $A$, whose objects are triples $(B, A, \phi)$, and morphisms $f:(B, A, \phi) \rightarrow\left(B^{\prime}, A, \phi^{\prime}\right)$ are $R$ linear maps $f: B \rightarrow B^{\prime}$ satisfying the relation $\left(I_{A} \otimes f\right) \circ \phi=$ $\phi^{\prime} \circ\left(f \otimes I_{A}\right)$. Then, there exist the following functors.

(1) $F: \operatorname{Mod} A \rightarrow$ SemiEntwining Str $A$.

$M \mapsto(M, A, \phi)$, where $\phi: M \otimes A \rightarrow A \otimes M, \phi(m \otimes$ a) $=1 \otimes m a$ 
(2) G: SemiEntwining $\operatorname{Str} A \rightarrow \operatorname{Mod} A$.

$(B, A, \psi) \mapsto A \otimes B$, where $A \otimes B$ is a right $A$-module with the operation $(a \otimes b) * a^{\prime}=a a_{\alpha}^{\prime} \otimes b^{\alpha}$.

These two functors do not form an equivalence of categories in general, because $F \circ G \simeq A \otimes-$ and $G \circ F \simeq A \otimes-$.

Theorem 9. If $\psi: B \otimes A \rightarrow A \otimes B$ is a semientwining map, and $A$ is bialgebra, then

(1) B is an A-bimodule with the following actions:

$a \circ b=\epsilon(a) b, \quad b * a=\epsilon\left(a_{\alpha}\right) b^{\alpha}, \quad \forall a \in A, \forall b \in B$.

(2) $B \oplus A$ is an algebra with the unit $(0,1)$ and the product

$$
(b, a)\left(b^{\prime}, a^{\prime}\right)=\left(b * a^{\prime}+a \circ b^{\prime}, a a^{\prime}\right)
$$

and a right $A$-comodule with the coaction $b \oplus a \mapsto b \otimes$ $1+\left(\sum a_{1} \otimes a_{2}\right)$.

(3) If $A$ has a bilateral integral (i.e.,ax $=x a=\epsilon(a) x$ $\forall a \in A$ ) which is a group-like element (i.e., $\Delta(x)=$ $x \otimes x, \epsilon(x)=1)$, then $B \oplus A$ is an $A$-comodule algebra with the coaction

$$
b \oplus a \longmapsto b \otimes x+\left(\sum a_{1} \otimes a_{2}\right)
$$

Proof. (1) Follows from the linearity of $\epsilon$ and $\psi$.

(2) Follows from the previous statement and from direct computations as follows: $b \oplus a \mapsto b \otimes 1+\left(\sum a_{1} \otimes a_{2}\right)$ maps to either $b \otimes(1 \otimes 1)+\left(\sum a_{1} \otimes\left(a_{21} \otimes a_{22}\right)\right)$ (if we apply the comultiplication of the algebra), or to $(b \otimes 1) \otimes 1+\left(\sum 0 \otimes 0\right) \otimes$ $1+0 \otimes 1+\left(\sum\left(a_{11} \otimes a_{12}\right) \otimes a_{2}\right)$ (if we apply the coaction).

We observe that the two outputs are equal.

(3) Is a generalisation of (2) and is left to the reader.

Similarly we have the dual notion as follows.

Definition 10 (cosemientwining structures). Let $C$ be an $R$ coalgebra, and let $D$ be an $R$-module. A $R$-linear map $\psi: D \otimes$ $C \rightarrow C \otimes D$ is called a cosemientwining map if it satisfies the following conditions for all $c, c^{\prime} \in C, d \in D$ (where we use a Sweedler-like summation notation $\left.\psi(d \otimes c)=c^{\alpha} \otimes d_{\alpha}\right)$ :

$$
\begin{gathered}
\varepsilon\left(c^{\alpha}\right) d_{\alpha}=\varepsilon(c) d, \\
c_{(1)}^{\alpha} \otimes c^{\alpha}{ }_{(2)} \otimes d_{\alpha}=c_{(1)}{ }^{\alpha} \otimes c_{(2)}{ }^{\beta} \otimes d_{\alpha \beta} .
\end{gathered}
$$

If $D$ is also a coalgebra, and $\psi$ satisfies additionally

$$
\begin{aligned}
c^{\alpha} \mathcal{E}\left(d_{\alpha}\right)=c \mathcal{E}(d), & \\
c^{\alpha} \otimes d_{\alpha(1)} \otimes d_{\alpha(2)}= & c^{\alpha \beta} \otimes d_{(1) \beta} \otimes d_{(2) \alpha} \\
& \forall d \in D, \quad \forall c \in C,
\end{aligned}
$$

then $\psi$ is called a coalgebra factorization.
If, on the other hand, $D$ is an algebra, and $\psi$ satisfies additionally

$$
\begin{array}{r}
\psi\left(1_{D} \otimes c\right)=c \otimes 1_{D}, \quad c^{\alpha} \otimes\left(d d^{\prime}\right)_{\alpha}=c^{\alpha \beta} \otimes d_{\beta} d_{\alpha}^{\prime}, \\
\forall d, d^{\prime} \in D, \forall c \in C,
\end{array}
$$

then $\psi$ is called a (right-right) entwining map.

The next result is dual to Lemma 5.

Lemma 11. Suppose that $\psi: D \otimes C \rightarrow C \otimes D$ is a cosemientwining map, and $D$ is a coalgebra. Define a map

$$
\begin{aligned}
& \Delta_{D \otimes C}:(D \otimes C) \longrightarrow(D \otimes C) \otimes(D \otimes C), \\
& d \otimes c \longmapsto\left(d_{(1)} \otimes c_{(1)}^{\alpha}\right) \otimes\left(d_{(2)_{\alpha}} \otimes c_{(2)}\right) .
\end{aligned}
$$

Then $\Delta$ makes $D \otimes C$ a coalgebra if and only if $\psi$ is a coalgebra factorization.

Proof. For $D \otimes C$ to be a coalgebra it must satisfy the counit property, that is, $\left(\varepsilon_{D \otimes C} \otimes \mathrm{id}\right) \circ \Delta_{D \otimes C}=\left(\mathrm{id} \otimes \varepsilon_{D \otimes C}\right) \circ \Delta_{D \otimes C}=\mathrm{id}$ and the coassociativity property. To check a counit property note that for all $d \in D$ and $c \in C$ :

$$
\left(\mathrm{id} \otimes \varepsilon_{D \otimes C}\right) \circ \Delta_{D \otimes C}(d \otimes c)=d_{(1)} \otimes c^{\alpha} \varepsilon\left(d_{(2)_{\alpha}}\right) .
$$

Now, if $d \otimes c=d_{(1)} \otimes c^{\alpha} \mathcal{E}\left(d_{(2)_{\alpha}}\right)$, then applying $\varepsilon \otimes$ id to both sides of this equation yields $c \varepsilon(d)=c^{\alpha} \varepsilon\left(d_{\alpha}\right)$. Similarly, we prove the other half of the counit property. Conversely, $c \varepsilon(d)=c^{\alpha} \varepsilon\left(d_{\alpha}\right)$ implies the counit property.

Using the fact that $\psi$ is a cosemientwining map, it is easy to prove that the coassociativity implies that for all $c \in C$ and $d \in D$

$$
c_{(1)}^{\alpha} \otimes d_{\alpha(1)} \otimes c_{(2)}^{\beta} \otimes d_{\alpha(2) \beta}=c_{(1)}^{\alpha \gamma} \otimes d_{(1) \gamma} \otimes c_{(2)}^{\beta} \otimes d_{(2) \alpha \beta} .
$$

Applying $\varepsilon$ to the third leg and using the fact that $\psi$ is a cosemientwining map yields

$$
c^{\alpha} \otimes d_{\alpha(1)} \otimes d_{\alpha(2)}=c^{\alpha \gamma} \otimes d_{(1) \gamma} \otimes d_{(2) \alpha} .
$$

We leave the rest of the proof to the reader.

Remark 12. Suppose that $C$ is a right $H$-comodule coalgebra (where $H$ is a bialgebra), and $D$ is a right $H$-module. Then

$$
\psi: D \otimes C \longrightarrow C \otimes D, \quad d \otimes c \longmapsto c_{(0)} \otimes d c_{(1)}
$$

is a cosemientwining map. Furthermore, if $D$ is an $H$-module coalgebra, then $\psi$ is a coalgebra factorization. Otherwise, if $D$ is an $H$-module algebra, then $\psi$ is a left-left entwining map. Moreover, in this last case, $(C, H, D)$ is called an alternative Doi-Koppinen structure.

Let $X, Y$ be any $R$-modules. Any $x^{*} \in X^{*}$ can be viewed as the map

$$
x^{*}: X \otimes Y \longrightarrow Y, \quad x \otimes y \longmapsto x^{*}(x) y .
$$


Also any tensor $\sum_{i} x_{i}^{*} \otimes y_{i} \in X^{*} \otimes Y$ can be considered as a map $X \ni x \mapsto \sum_{i} x_{i}^{*}(x) y_{i} \in Y$. Finally, if $X$ is finitely generated and projective, then $\operatorname{Hom}_{R}(X, Y) \sim X^{*} \otimes Y$. For any $y \in Y$, an $R$-module map $\Psi: Y \otimes X \rightarrow X \otimes Y$ defines a map

$$
\Psi_{y}=\Psi(y \otimes \cdot): X \longrightarrow X \otimes Y .
$$

We define a dual of $\Psi^{*} X: Y \otimes X^{*} \rightarrow X^{*} \otimes Y$ with respect to the $X$-part as $\Psi^{*} x\left(y \otimes x^{*}\right)=\Psi_{y}^{*}\left(x^{*}\right)$, where $\Psi_{y}^{*}: X^{*} \rightarrow$ $X^{*} \otimes Y$ is defined by

$$
x^{*}\left(\Psi_{y}(x)\right)=\psi_{y}^{*}\left(x^{*}\right)(x), \quad \forall x \in X, x^{*} \in X^{*}, y \in Y .
$$

Similarly, one defines a dual $\Psi^{*}: Y^{*} \otimes X \rightarrow X \otimes Y^{*}$ of $\Psi$ with respect to the $Y$-part.

The next lemma is a standard result.

Lemma 13. Suppose that $C$ is a finitely generated projective $R$ coalgebra, and $\left(c_{i} \in C, c_{i}^{*}\right)$ is a dual basis. Let $\psi: D \otimes C \rightarrow C \otimes$ $D$ be a cosemientwining map. Then $\psi^{{ }^{*} C}: D \otimes C^{*} \rightarrow C^{*} \otimes D$ is a semientwining map for the convolution algebra $C^{*}$.

Explicitly,

$$
\psi^{*} C\left(d \otimes c^{*}\right)=\sum_{i} c_{i}^{*} \otimes c^{*}\left(c_{i}^{\alpha}\right) d_{\alpha} .
$$

Definition 14 (semientwined modules and comodules). Let $A$ be an algebra, and let $V$ be a vector space. Suppose that $\psi: V \otimes A \rightarrow A \otimes V$ is a semientwining map, and $M$ a right $A$ module.

(1) Let $\triangleleft: M \otimes V \rightarrow M$ be a right measuring, such that for all $m \in M, a \in A, v \in V$,

$$
m a_{\alpha} \triangleleft v^{\alpha}=(m \triangleleft v) a .
$$

Then $M$ is called a $(A, V, \psi)$ semientwined module.

(2) Let $\rho: M \rightarrow M \otimes V, m \rightarrow m_{(0)} \otimes m_{(1)}$ be a right comeasuring, such that for all $m \in M, a \in A$,

$$
\rho(m a)=m_{(0)} \psi\left(m_{(1)} \otimes a\right) .
$$

Then $M$ is called a $(A, V, \psi)$ semientwined comodule.

Remark 15. The following are examples of semientwining modules related to Remark 4:

(1) let $A$ be an $R$-algebra, let $M$ be a right $A$ module, $V=$ $A, \psi=\gamma_{q}$, and the right measuring the regular action of $A$ on $M$;

(2) let $A$ be an $R$-algebra, let $M$ be a right $A$ module, $V=$ $A, \psi=\eta_{1}$, and the right measuring the regular action of $A$ on $M$.

Remark 16. The following are examples of semientwining comodules related to Remark 4:

(1) let $A$ be an $R$-algebra, let $M$ be a right $A$ module, $V=$ $A, \psi=\gamma_{1}$, and the right comeasuring $\rho(m)=m \otimes 1$;
(2) let $A$ be an $R$-algebra, let $M$ be a right $A$ module, $V=$ $A, \psi=\eta_{q}$, and the right comeasuring $\rho(m)=m \otimes 1$.

Definition 17 (cosemientwined modules and comodules). Let $C$ be a coalgebra, and let $V$ be a vector space. Suppose that $\psi: V \otimes C \rightarrow C \otimes V$ is a cosemientwining map, and $M$ a left $C$-comodule, with a coaction ${ }^{C} \rho: M \rightarrow C \otimes M$, $m \mapsto m_{(-1)} \otimes m_{(0)}$.

(1) Let $\triangleright: V \otimes M \rightarrow M$ be a left measuring, such that for all $m \in M, v \in V$,

$$
{ }^{C} \rho(v \triangleright m)=m_{(-1)_{\alpha}} \otimes v^{\alpha} \triangleright m_{(0)} .
$$

Then $M$ is called a $(C, V, \psi)$ cosemientwined module.

(2) Let ${ }^{V} \rho: M \rightarrow V \otimes M, m \mapsto m_{-1} \otimes m_{\overline{0}}$ be a left comeasuring, such that for all $m \in M$,

$$
\left(\mathrm{id}_{C} \otimes{ }^{V} \rho\right){ }^{C} \rho(m)=m_{\overline{0}(-1) \alpha} \otimes m_{-1}^{\alpha} \otimes m_{\overline{0}(0)} .
$$

Then $M$ is called a $(C, V, \psi)$ cosemientwined comodule.

Note that if $V$ is a coalgebra, and $\psi: V \otimes A \rightarrow A \otimes V$ is an entwining map, then a semientwined module $M$ is an entwined module.

The following result is standard, but we provide a partial proof for completeness.

Lemma 18. Suppose that $(A, B, \psi)$ is an algebra factorization, and $M$ is a $(A, B, \psi)$ semientwined module, such that the $B$ measuring is an action. Then $M$ is a right $A \otimes B$-module, with an algebra structure on $A \otimes B$ as in Lemma 5 , and $A \otimes B$ action on $M$ given by $m(a \otimes b)=(m a) \triangleleft b$. Conversely, any right $A \otimes B$ module is a semientwined $(A, B, \psi)$ module with $A$ and $B$ actions given by $m a=m\left(a \otimes 1_{B}\right)$ and $m \triangleleft b=m\left(1_{A} \otimes b\right)$, respectively.

Proof. It is enough to verify that the definition of $A \otimes B$ action agrees with the algebra relations, that is, that

$$
m((1 \otimes b)(a \otimes 1))=(m(1 \otimes b))(a \otimes b) .
$$

Both sides of the above equation equal $m a_{\alpha} \triangleleft b^{\alpha}$-left one because of algebra relations, and the right one because $M$ is a $(A, B, \psi)$ semientwined module. We prove similarly the rest of the lemma.

\section{Applications}

3.1. Intertwining Operators. We give a brief introduction to the intertwining operators below.

Let $A$ be an $R$-algebra. Given two algebra representations, say $\rho: V \otimes A \rightarrow V$ and $\rho^{\prime}: V^{\prime} \otimes A \rightarrow V^{\prime}$, we define an intertwining operator $f: V \rightarrow V^{\prime}$ to be a linear operator, such that $f \circ \rho=\rho^{\prime} \circ(f \otimes I)$.

With this definition we can define the category of finitedimensional representations of $A$, in which the morphisms are intertwining operators (see [18]). 
The following theorem provides a connection between semientwining structures and intertwining operators.

Theorem 19. Let $A$ be an $R$-algebra, let $B$ be an $R$-module, and let $\psi: B \otimes A \rightarrow A \otimes B$ be a semientwining map. Then, the following statements are true:

(i) $B \otimes A$ is a right $A$-module in a trivial way, with the right action $\rho:(B \otimes A) \otimes A \rightarrow(B \otimes A),(b \otimes a) \otimes a^{\prime} \mapsto$ $b \otimes a a^{\prime}$.

(ii) $A \otimes B$ is a right $A$-module in the following way: $\rho^{\prime}$ : $(A \otimes B) \otimes A \rightarrow(A \otimes B),(a \otimes b) \otimes a^{\prime} \mapsto a a_{\alpha}^{\prime} \otimes b^{\alpha}$.

(iii) With the above actions, $\psi: B \otimes A \rightarrow A \otimes B$ is an intertwining operator (i.e., $\psi$ satisfies the relation $\psi \circ$ $\left.\rho=\rho^{\prime} \circ(\psi \otimes I)\right)$.

Proof. The proof of (i) is direct, and (ii) follows from Lemma 5(i). The relation $\psi \circ \rho=\rho^{\prime} \circ(\psi \otimes I)$ is equivalent to the second relation of (3).

3.2. Braided Algebras. Many algebras obtained by quantization are commutative braided algebras, and all super-commutative algebras are automatically commutative braided algebras (see [19]).

Definition 20. An algebra $(A, M, u)$ for which there exists a Yang-Baxter operator $\psi: A \otimes A \rightarrow A \otimes A$ such that $\psi(a \otimes 1)=$ $1 \otimes a, \psi(1 \otimes b)=b \otimes 1, \psi(a \otimes b c)=(M \otimes I) \circ(I \otimes \psi) \circ(\psi \otimes$ $I)(a \otimes b \otimes c)$, and $\psi(a b \otimes c)=(I \otimes M) \circ(\psi \otimes I) \circ(I \otimes \psi)(a \otimes$ $b \otimes c)$ for all $a, b, c \in A$ is called a braided algebra.

Moreover, if $M \circ \psi(a \otimes b)=M(a \otimes b)$ for all $a, b \in A$, we call $(A, M, u, \psi)$ a commutative braided algebra or an $r$ commutative algebra (see [20]).

Definition 21. Given braided algebras $(A, M, u, \psi)$ and $\left(B, M, u, \psi^{\prime}\right)$, we say that $f: A \rightarrow B$ is a braided algebra morphism if it is a morphism of algebras and $(f \otimes f) \circ \psi=$ $\psi^{\prime} \circ(f \otimes f)($ see $[20])$.

Theorem 22. (i) Any algebra $(A, M, u)$ becomes a commutative braided algebra $(A, M, u, \psi)$ with $\psi(a \otimes b)=\psi^{A}(a \otimes b)=$ $1 \otimes a b+a b \otimes 1-a \otimes b$.

(ii) If $\left(A, M, u, \psi^{A}\right)$ and $\left(B, M, u, \psi^{B}\right)$ are two braided algebras as in (i), and $f: A \rightarrow B$ is an algebra morphism, then it is also a braided algebra morphism.

(iii) If $\delta: A \rightarrow A$ is a derivation (i.e., $\delta(a b)=\delta(a) b+$ $a \delta(b)$ and $\delta(1)=0)$, then there exists a morphism of braided algebras $f:\left(A, M, u, \psi^{A}\right) \rightarrow\left(A \oplus A, m, \eta, \psi^{A \oplus A}\right), a \mapsto$ $a \oplus \delta(a)$, where $m\left((a \oplus b) \otimes\left(a^{\prime} \oplus b^{\prime}\right)\right)=\left(a a^{\prime}\right) \oplus\left(a b^{\prime}+\right.$ ba') and $1_{A \oplus A}=1_{A} \oplus 0_{A}$.

Proof. (i) Notice that $\psi(a \otimes b)=1 \otimes a b+a b \otimes 1-a \otimes b$ is a selfinverse Yang-Baxter operator which was studied in $[16,21]$.

\begin{abstract}
$\psi(a \otimes 1)=1 \otimes a, \psi(1 \otimes b)=b \otimes 1$ (directly)
$\psi(a \otimes b c)=(M \otimes I) \circ(I \otimes \psi) \circ(\psi \otimes I)(a \otimes b \otimes c)$ (from Remark 4 (i) with $q=1$ )

$\psi(a b \otimes c)=1 \otimes a b c+a b c \otimes 1-a b \otimes c=$ $(I \otimes M) \circ(\psi \otimes I) \circ(I \otimes \psi)(a \otimes b \otimes c)=(I \otimes M) \circ$ $(\psi \otimes I)(a \otimes 1 \otimes b c+a \otimes b c \otimes 1-a \otimes b \otimes c)=$ $(I \otimes M)(1 \otimes a \otimes b c+a b c \otimes 1 \otimes 1+1 \otimes a b c \otimes$ $1-a \otimes b c \otimes 1-1 \otimes a b \otimes c-a b \otimes 1 \otimes c+a \otimes b \otimes c)=$ $1 \otimes a b c+a b c \otimes 1+1 \otimes a b c \otimes 1-a \otimes b c-1 \otimes$ $a b c-a b \otimes c+a \otimes b c=a b c \otimes 1-1 \otimes a b c-a b \otimes c$ $M \circ \psi(a \otimes b)=1 \otimes a b+a b \otimes 1-a \otimes b=a b=M(a \otimes b)$
\end{abstract}

(ii) This follows from Proposition 3.1 of [15]. Also, refer to [16].

(iii) The proof is direct and is left to the reader.

Remark 23. In the above example $\psi \circ \psi=I \otimes I$; so, the above algebra is "strong." All sorts of noncommutative analogs of manifolds are commutative braided algebras: quantum groups, noncommutative tori, quantum vector spaces, the Weyl and Clifford algebras, certain universal enveloping algebras, super-manifolds, and so forth. It seems that the ones with direct relevance to quantum theory in 4 dimensions are "strong," while the nonstrong ones, like quantum groups, are primarily relevant to 2- and 3-dimensional physics (see [19]).

3.3. Liftings of Functors. The semientwining structures can be understood as liftings of functors from one category to another. This goes back as far back as [22]. This situation is reviewed in [11]: the semientwining case is dealt with in general in item 3.3 (which is transferred from [22]); how this general case is translated to our situation is clear from the discussion in item 5.8 of [11]. This is also presented in Section 3.1 of [23], where the axioms of semientwining structures are given by formula (3.1).

We give a general definition of liftings of functors. $F$ is a lifting of $G$ if the following diagram commutes

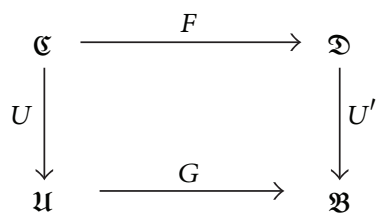

where $U$ and $U^{\prime}$ are forgetful functors.

We now present examples of liftings of functors related to semientwining structures.

Theorem 24. Let $A$ be an $R$-algebra, and let $B$ be an $R$-module. The functor $-\otimes B$ can be lifted from the category of $R$-modules to the category of right $A$-modules $\Leftrightarrow$ there exists a $R$-linear map $\psi: B \otimes A \rightarrow A \otimes B$ which is a semientwining map.

Proof. Assume that there exists a semientwining $\psi: B \otimes$ $A \rightarrow A \otimes B$, then $-\otimes B$ lifts to a functor which associates to a right $A$-module $M$ the $A$-module $M \otimes B$ with a right $A$ action given by

$$
(m \otimes b) a:=m a_{\alpha} \otimes b^{\alpha} .
$$


It remains to check that for any right $A$-module function $f$ : $M \rightarrow M^{\prime}$, the map $f \otimes$ id $: M \otimes B \rightarrow M^{\prime} \otimes B$ is a right $A$-module map as follows:

$$
\begin{aligned}
(f \otimes \mathrm{id})(m \otimes b) a & =(f(m) \otimes b) a=f(m) a_{\alpha} \otimes b^{\alpha} \\
& =f\left(m a_{\alpha}\right) \otimes b^{\alpha}=(f \otimes \mathrm{id})((m \otimes b) a) .
\end{aligned}
$$

On the other hand, suppose that $-\otimes B$ lifts to a functor in the category of right $A$-modules. In particular, it follows that $A \otimes B$ is a right $A$-module. Define the linear map

$$
\Psi: B \otimes A \longrightarrow A \otimes B, \quad b \otimes a \longmapsto a_{\alpha} \otimes b^{\alpha}
$$

by the formula

$$
\Psi(b \otimes a):=(1 \otimes b) a .
$$

We shall prove that this is a semientwining map. Indeed, by definition we have

$$
\Psi(b \otimes 1)=1 \otimes b .
$$

Any element $a \in A$ defines a right $A$-module map

$$
f: A \longrightarrow A, \quad a^{\prime} \longmapsto a a^{\prime} .
$$

It follows that for any $a^{\prime} \in A$, we have from the $A$-linearity of $f \otimes \mathrm{id}$ as follows:

$$
\begin{aligned}
(a \otimes b) a^{\prime} & =(f(1) \otimes b) a^{\prime}=(f \otimes \mathrm{id})\left((1 \otimes b) a^{\prime}\right) \\
& =f\left(a_{\alpha}^{\prime}\right) \otimes b^{\alpha}=a a_{\alpha}^{\prime} \otimes b^{\alpha} .
\end{aligned}
$$

Hence $\left(a a^{\prime}\right)_{\alpha} \otimes b^{\alpha}=(1 \otimes b)\left(a a^{\prime}\right)=\left(a_{\alpha} \otimes b^{\alpha}\right) a^{\prime}=a_{\alpha} a_{\beta}^{\prime} \otimes$ $b^{\alpha \beta}$.

Remark 25. Let $A$ be an $R$-algebra, and let $B$ be an $R$-module. Using our terminology (given in Remark 8) and the results of [23], we conclude that the category of semientwining structures over $A$ is isomorphic to the category of lifting of functors from the category of $R$-modules to the category of right $A$-modules.

Remark 26. We now give a more general definition than that given in Remark 8.

We define the category of semientwining structures, whose objects are triples $(B, A, \phi)$, and morphisms are pairs $(f, g):(B, A, \phi) \rightarrow\left(B^{\prime}, A^{\prime}, \phi^{\prime}\right)$ where $f: B \rightarrow B^{\prime}$ is an $R$-linear map, $g: A \rightarrow A^{\prime}$ is an algebra morphism, and they satisfy the relation $(g \otimes f) \circ \phi=\phi^{\prime} \circ(f \otimes g)$.

In a dual manner, let us define the category of cosemientwining structures, whose objects are triples $(D, C, \phi)$, and morphisms are pairs $(f, g):(D, C, \phi) \rightarrow\left(D^{\prime}, C^{\prime}, \phi^{\prime}\right)$ where $f: D \rightarrow D^{\prime}$ is an $R$-linear map, $g: C \rightarrow C^{\prime}$ is a coalgebra morphism, and they satisfy the relation $(g \otimes f) \circ \phi=$ $\phi^{\prime} \circ(f \otimes g)$.
The duality functor from the category of coalgebras to the category of algebras can be lifted to a functor from the category of cosemientwining structures to the category of semientwining structures (by Lemma 13).

This fact is described in the following diagram:

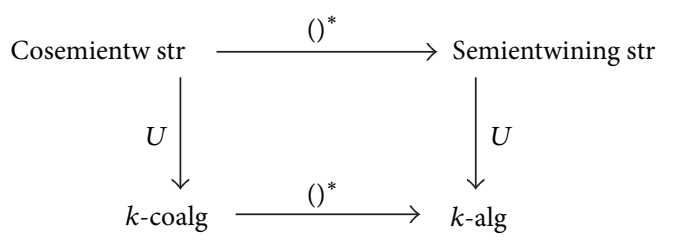

Remark 27. A braided coalgebra is a structure dual to Definition 20 (see, e.g. [24]).

The duality between finite-dimensional algebras and finite-dimensional coalgebras can be lifted to a duality between the categories of finite-dimensional-braided algebras and finite- dimensional braided coalgebras. This fact is described in the following diagram:

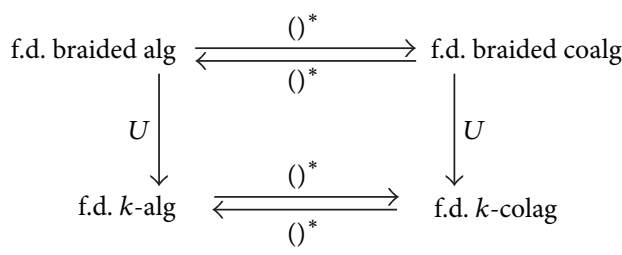

\subsection{Tambara Bialgebras.}

Definition 28 (Tambara bialgebra [25]). Let $A$ be a finitely generated and projective $R$-algebra (which implies that $A^{*}$ is a coalgebra), and let $a_{i}, a_{i}^{*}, i=1, \ldots, N$ be a dual basis of $A$. Let $I \subset T\left(A^{*} \otimes A\right)$ be an ideal generated by elements

$$
\begin{gathered}
a^{*}\left(1_{A}\right)-a^{*} \otimes 1_{A}, \\
a^{*} \otimes a a^{\prime}-a^{*}{ }_{(1)} \otimes a \otimes a^{*}{ }_{(2)} \otimes a^{\prime},
\end{gathered}
$$

for all $a \in A, a^{*} \in A^{*}$. Then $H(A)=T\left(A^{*} \otimes A\right) / I$ is called a Tambara bialgebra. Denoting by $\left[a^{*} \otimes a\right]$ the class of $a \otimes a^{*}$ in $H(A)$, the comultiplication $\Delta$ and counit $\varepsilon$ is given by

$$
\begin{gathered}
\Delta\left(\left[a^{*} \otimes a\right]\right)=\left[\sum_{i} a^{*} \otimes a_{i}\right] \otimes\left[a_{i}^{*} \otimes a\right], \\
\varepsilon\left(\left[a^{*} \otimes a\right]\right)=a^{*}(a) .
\end{gathered}
$$

$A$ is a right $H(A)$-comodule algebra with coaction

$$
\varrho(a)=\sum_{i} a_{i} \otimes\left[a_{i}^{*} \otimes a\right] .
$$

Theorem 29 (see [25]). Suppose that $A$ is a finitely generated projective $R$-algebra, and $B$ is an $R$-module. Then semientwining structures $\psi: B \otimes A \rightarrow A \otimes B$ are in one-toone correspondence with right $H(A)$-module structures on $B$. Similarly, if $B$ is an algebra, then algebra factorizations are in 
one to one correspondence with right $H(A)$-module algebra structures on $B$. Finally if $B$ is a coalgebra, then entwining structures $\psi: B \otimes A \rightarrow A \otimes B$ are in one to one correspondence with right $H(A)$-module coalgebra structures on $B$. Explicitly, given right $H(A)$-module structure on $B$, we define $\psi=\psi_{H(A)}$ (7). Conversely, given a semientwining $\psi: B \otimes A \rightarrow A \otimes B$, we define a right $H(A)$ module action on $B$ by

$$
b\left[a^{*} \otimes a\right]=a^{*}\left(a_{\alpha}\right) b^{\alpha} .
$$

Remark 30. Let $q \in R$. The examples of semientwining structures presented in Remark 4 generate the following structures:

(1) a right $H(A)$ module action on $A$ by

$$
b\left[a^{*} \otimes a\right]=a^{*}(1) b a+q a^{*}(b a) 1_{A}-q a^{*}(b) a ;
$$

(2) a right $H(A)$ module action on $A$ by

$$
b\left[a^{*} \otimes a\right]=q a^{*}(b a-a b) 1_{A}+q a^{*}(a) b ;
$$

(3) a right $H(A)$ module action on $M$, for any right $A$ module $M$, by

$$
m\left[a^{*} \otimes a\right]=a^{*}\left(1_{A}\right) m a .
$$

Let $C$ be a finitely generated and projective $R$ coalgebra. Let $c_{i}, c_{i}^{*}, i=1, \ldots, N$ be a dual basis of $C$. Note that $H\left(C^{*}\right)^{\text {cop }}=T\left(C^{*} \otimes C\right) / I^{\prime}$ where $I^{\prime} \subset T\left(C^{*} \otimes C\right)$ is an ideal generated by elements

$$
\begin{gathered}
\varepsilon_{C}(c)-\varepsilon_{C} \otimes C, \\
c^{*} * d^{*} \otimes c-\left(c^{*} \otimes c_{(1)}\right) \otimes\left(d^{*} \otimes c_{(2)}\right),
\end{gathered}
$$

for all $c^{*}, d^{*} \in C^{*}, c \in C$, with explicit coaction and counit given by

$$
\begin{gathered}
\Delta\left(\left[c^{*} \otimes c\right]\right)=\left[\sum_{i} c^{*} \otimes c_{i}\right] \otimes\left[c_{i}^{*} \otimes c\right] \\
\varepsilon\left(\left[c^{*} \otimes c\right]\right)=c^{*}(c)
\end{gathered}
$$

Theorem 31 (see [25]). Suppose that $C$ is a finitely generated projective $R$-coalgebra, and $D$ is an $R$-module. Then cosemientwining structures $\psi: D \otimes C \rightarrow C \otimes D$ are in one-to-one correspondence with right $H\left(C^{*}\right)^{\text {cop }}$ module structures on $D$. Similarly if $D$ is a coalgebra, then coalgebra factorizations are in one to one correspondence with $H\left(C^{*}\right)^{\text {cop }}$ module coalgebra structures on $D$. Finally, if $D$ is an algebra, then (right-right) entwining structures $\psi: D \otimes C \rightarrow C \otimes D$ are in one to one correspondence with right $H\left(C^{*}\right)^{\text {cop }}$-module algebra structures on $D$. Explicitly, given right $H\left(C^{*}\right)^{\text {cop }}$ module structures on $D$, we define $\psi=\psi^{H\left(C^{*}\right)^{\text {cop }}}$ (18). Conversely, given a cosemientwining $\psi: D \otimes C \rightarrow C \otimes D$, we define a right $H\left(C^{*}\right)^{\mathrm{cop}}$-module structures on $D$ by

$$
d\left[c^{*} \otimes c\right]=c^{*}\left(d_{\alpha}\right) c^{\alpha}
$$

3.5. Yang-Baxter Systems. From now on we work over a field $\mathbb{K}$. It is convenient to introduce the constant Yang-Baxter commutator of the linear maps $R: V \otimes V^{\prime} \rightarrow V \otimes V^{\prime}, S:$ $V \otimes V^{\prime \prime} \rightarrow V \otimes V^{\prime \prime}, T: V^{\prime} \otimes V^{\prime \prime} \rightarrow V^{\prime} \otimes V^{\prime \prime}$ by

$$
[R, S, T]:=R_{12} S_{13} T_{23}-T_{23} S_{13} R_{12} \text {. }
$$

In this notation, the quantum Yang-Baxter equation reads $[R, R, R]=0$.

Definition 32 (Yang-Baxter systems of type I). A system of linear maps of vector spaces $W: V \otimes V \rightarrow V \otimes V, Z$ : $V^{\prime} \otimes V^{\prime} \rightarrow V^{\prime} \otimes V^{\prime}, X: V \otimes V^{\prime} \rightarrow V \otimes V^{\prime}$ is called a $W X Z$ system (or a Yang-Baxter system of type I) if

$$
\begin{array}{rlrl}
{[W, W, W]} & =0, & {[W, X, X]} & =0, \\
{[Z, Z, Z]} & =0, & {[X, X, Z]=0 .}
\end{array}
$$

A system of linear maps $W, X$ satisfying (49) is called a semi Yang-Baxter system. One can associate a $W X Z$ system to a semi Yang-Baxter system by setting $Z=I \otimes I$.

Remark 33. From a Yang-Baxter system of type I, one can construct a Yang-Baxter operator on $(V \oplus V) \otimes(V \oplus V)$, provided that the map $X$ is invertible (see [6]).

Let $A$ be an algebra, and the map

$$
\begin{aligned}
& W=R_{r, s}^{A}: A \otimes A \longrightarrow A \otimes A, \\
& a \otimes b \longmapsto s b a \otimes 1+r 1 \otimes b a-s b \otimes a,
\end{aligned}
$$

for some arbitrary $s, r \in \mathbb{K}$ (see [15]). Then, $[W, W, W]=0$.

The following is an enhanced version of Theorem 2.3 of [6].

Theorem 34 (see [6]). Let $A$ be an algebra, let $B$ be a vector space, and $p, q, s, r \in \mathbb{K}$.

Let $W=R_{r, s}^{A}$, and let $X: A \otimes B \rightarrow A \otimes B$ be a linear map, such that $X\left(1_{A} \otimes b\right)=1_{A} \otimes b$, for all $b \in B$.

(i) Then $W, X$ is a semi Yang-Baxter system if and only if $\psi=X \circ \tau_{B, A}$ is a semientwining map.

(ii) Similarly, if $B$ is an algebra, $Z=R_{p, q}^{B}$, and $X(a \otimes$ $\left.1_{B}\right)=a \otimes 1_{B}$, for all $a \in A$, then $W, X, Z$ is a YangBaxter system of type I if and only if $\psi$ is an algebra factorization.

Definition 35 (Yang-Baxter systems of type II). A system of linear maps of vector spaces $\mathbb{A}, \mathbb{B}, \mathbb{C}, \mathbb{D}: V \otimes V \rightarrow V \otimes V$ is called a Yang-Baxter system of type II if

$$
\begin{aligned}
{[\mathbb{A}, \mathbb{A}, \mathbb{A}] } & =0, & & {[\mathbb{D}, \mathbb{D}, \mathbb{D}]=0, } \\
{[\mathbb{A}, \mathbb{C}, \mathbb{C}] } & =0, & & {[\mathbb{D}, \mathbb{B}, \mathbb{B}]=0, } \\
{\left[\mathbb{A}, \mathbb{B}^{+}, \mathbb{B}^{+}\right] } & =0, & & {\left[\mathbb{D}, \mathbb{C}^{+}, \mathbb{C}^{+}\right]=0, } \\
{\left[\mathbb{A}, \mathbb{C}, \mathbb{B}^{+}\right] } & =0, & & {\left[\mathbb{D}, \mathbb{B}, \mathbb{C}^{+}\right]=0, }
\end{aligned}
$$

where $X^{+}=\tau X \tau$ (and $\tau$ is the twist map). 
Remark 36. Yang-Baxter systems of type II are related to the algebras considered in [4], which include (algebras of functions on) quantum groups, quantum super-groups, braided groups, quantized braided groups, reflection algebras, and others.

The following theorems present solutions for the YangBaxter systems.

Theorem 37 (see [9]). Let $A$ be a commutative algebra, and $\lambda, \lambda^{\prime} \in \mathbb{K}$. Then, $\mathbb{A}, \mathbb{B}, \mathbb{C}, \mathbb{D}: A \otimes A \rightarrow A \otimes A, \mathbb{A}(a \otimes b)=$ $\lambda 1 \otimes a b+a b \otimes 1-b \otimes a, \mathbb{B}(a \otimes b)=\mathbb{C}(a \otimes b)=1 \otimes a b+$ $a b \otimes 1-b \otimes a$ and $\mathbb{D}(a \otimes b)=\lambda^{\prime} 1 \otimes a b+a b \otimes 1-b \otimes a$ is a Yang-Baxter system of type II.

Theorem 38. Let $W=\mathbb{A}, X=\mathbb{B}=\mathbb{C}, Z=\mathbb{D}$ in the above theorem. It turns out that $W, X, Z$ is also a Yang-Baxter system of type I.

Proof. First, let us observe that the result holds even for $A$ a noncommutative algebra. One way to prove the theorem is by direct computations.

Alternatively, one can observe that

$$
\psi(a \otimes b)=1 \otimes a b+a b \otimes 1-a \otimes b
$$

is an algebra factorization, and apply Remark 2.4 of [6].

Also, refer to Theorem 5.2 of [8].

Remark 39. One can combine the proof of the Theorem 38 with Remark 2.4 and Proposition 2.9 of [6] to obtain a large class of Yang-Baxter operators defined on $V \otimes V$, where $V=$ $A \oplus A$. See also Remark 33.

Theorem 40. Let $A$ be an algebra; $p, q, s, r \in \mathbb{K} ; \psi, \psi^{\prime}: A \otimes$ $A \rightarrow A \otimes A$ semientwining maps; $\mathbb{A}, \mathbb{B}, \mathbb{C}, \mathbb{D}: A \otimes A \rightarrow$ $A \otimes A, \mathbb{A}=R_{r, s}^{A}, \mathbb{B}=\psi \circ \tau, \mathbb{C}=\psi^{\prime} \circ \tau, \mathbb{D}=R_{p, q}^{A}$. If $\psi^{\prime}=\tau \circ \psi \circ \tau$, then $\mathbb{A}, \mathbb{B}, \mathbb{C}, \mathbb{D}$ is a Yang-Baxter system of type II.

Proof. Use Theorem 34 (i) to check the first-four equations. Then, observe that $\mathbb{B}=\mathbb{C}^{+} \Leftrightarrow \psi^{\prime}=\tau \circ \psi \circ \tau$. The last-four equations then follow.

Theorem 41. Let $A$ be an algebra, and $\psi: A \otimes A \rightarrow A \otimes A a$ semientwining map.

Then, there exists a semientwining map $\psi^{\prime}: A \otimes A \rightarrow$ $A \otimes A$, such that $\psi^{\prime}=\tau \circ \psi \circ \tau$ if and only if $\psi$, viewed as $\psi: \mathrm{A}^{\mathrm{op}} \otimes \mathrm{A} \rightarrow \mathrm{A} \otimes \mathrm{A}^{\mathrm{op}}$, is an algebra factorization.

Proof. Assume that there exists a semientwining map $\psi^{\prime}=$ $\tau \circ \psi \circ \tau$. Denote $\psi^{\prime}(a \otimes b)=b_{\alpha^{\prime}} \otimes a^{\alpha^{\prime}}$, for all $a, b \in A$, that is, $a_{\alpha} \otimes b^{\alpha}=a^{\alpha^{\prime}} \otimes b_{\alpha^{\prime}}$. Also denote by op the multiplication in $A^{\mathrm{op}}$, that is, for all $a, b \in A, a_{{ }_{\mathrm{op}}} b \equiv b a$. Then we must check conditions (4). For all $a, b, c \in A$,

$$
\begin{aligned}
\psi\left(1_{A^{\mathrm{op}}} \otimes c\right) & =\tau \circ \psi^{\prime} \circ \tau\left(1_{A^{\mathrm{op}}} \otimes c\right) \\
& =\tau \circ \psi^{\prime}\left(c \otimes 1_{A^{\mathrm{op}}}\right)=c \otimes 1_{A^{\mathrm{op}}},
\end{aligned}
$$

$$
\begin{aligned}
\psi\left(a{ }_{\mathrm{op}} b \otimes c\right) & =\tau \circ \psi^{\prime} \circ \tau(b a \otimes c) \\
& =\tau \circ \psi^{\prime}(c \otimes b a)=\tau\left(b_{\alpha^{\prime}} a_{\beta^{\prime}} \otimes c^{\alpha^{\prime} \beta^{\prime}}\right) \\
& =c^{\alpha^{\prime} \beta^{\prime}} \otimes a_{\beta^{\prime}{ }_{\mathrm{op}}} b_{\alpha^{\prime}}=c_{\alpha \beta} \otimes a^{\beta} \cdot{ }_{\mathrm{op}} b^{\alpha} .
\end{aligned}
$$

Similarly one can prove the converse.

Remark 42 (example of algebra factorization for Theorem 41). We consider the algebra $A=A^{\mathrm{op}}=\mathbb{K}[X] /\left(X^{2}-p\right)$, where $p$ is a scalar. Then $A$ has the basis $\{1, x\}$, where $x$ is the image of $X$ in the factor ring, so $x^{2}=p$. follows

If $q$ is a scalar, then $\psi: A^{\text {op }} \otimes A \rightarrow A \otimes A^{\text {op }}$, defined as

$$
\begin{gathered}
\psi(1 \otimes 1)=1 \otimes 1, \\
\psi(1 \otimes x)=x \otimes 1, \\
\psi(x \otimes 1)=1 \otimes x, \\
\psi(x \otimes x)=q 1 \otimes 1-x \otimes x,
\end{gathered}
$$

is an algebra factorization.

Notice that if $q=2 p$, then $\psi$ is the same algebra factorization with (53).

Theorem 43. Let $A$ be an algebra, let $B$ and $M$ be vector spaces, $z \in B(z \neq 0), \psi: B \otimes A \rightarrow A \otimes B$ a semientwining, and let $M$ be an $(A, B, \psi)$ semientwined module with the right measuring $\phi$. We consider the maps as follows:

$$
\begin{gathered}
X=\psi \circ \tau_{B, A}: B \otimes A \longrightarrow B \otimes A, \\
\eta: M \otimes A \longrightarrow M \otimes A, \quad m \otimes a \longmapsto m a \otimes 1_{A}, \\
\zeta: M \otimes B \longrightarrow M \otimes B, \quad m \otimes b \longmapsto \phi(m \otimes b) \otimes z .
\end{gathered}
$$

Then, the following equation holds

$$
[\zeta, \eta, X]=0 .
$$

Proof. The proof follows by direct computations.

Remark 44. The relation $[\zeta, \eta, X]=0$ from the above theorem is related to Section 3.6 of [23].

\section{References}

[1] M. Marcolli and D. Parashar, Quantum Groups and Noncommutative Spaces, Vieweg-Tuebner, 2010.

[2] T. Brzeziński and S. Majid, "Coalgebra bundles," Communications in Mathematical Physics, vol. 191, no. 2, pp. 467-492, 1998.

[3] J. Beck, "Distributive laws," in Seminar on Triples and Categorical Homology Theory, B. Eckmann, Ed., vol. 80 of Springer Lecture Notes in Mathematics, pp. 119-140, Springer, Berlin, Germany, 1969.

[4] L. Hlavatý, "Algebraic framework for quantization of nonultralocal models," Journal of Mathematical Physics, vol. 36, no. 9, pp. 4882-4897, 1995. 
[5] L. Hlavaty and L. Snobl, "Solution of a Yang-Baxter system," http://arxiv.org/abs/math/9811016.

[6] T. Brzeziński and F. F. Nichita, "Yang-Baxter systems and entwining structures," Communications in Algebra, vol. 33, no. 4, pp. 1083-1093, 2005.

[7] B. R. Berceanu, F. F. Nichita, and C. Popescu, "Algebra structures arising from Yang-Baxter systems," http://arxiv .org/abs/1005.0989.

[8] F. F. Nichita and D. Parashar, "Spectral-parameter dependent Yang-Baxter operators and Yang-Baxter systems from algebra structures," Communications in Algebra, vol. 34, no. 8, pp. 2713-2726, 2006.

[9] F. F. Nichita and D. Parashar, "New constructions of YangBaxter systems," AMS Contemporary Mathematics, vol. 442, pp. 193-200, 2007.

[10] F. F. Nichita and C. Popescu, "Entwined bicomplexes," Bulletin Mathématique de la Société des Sciences Mathématiques de Roumanie, vol. 52, no. 2, pp. 161-176, 2009.

[11] R. Wisbauer, "Algebras versus coalgebras," Applied Categorical Structures, vol. 16, no. 1-2, pp. 255-295, 2008.

[12] S. Caenepeel and K. Janssen, "Partial entwining structures," Communications in Algebra, vol. 36, no. 8, pp. 2923-2946, 2008.

[13] P. Schauenburg, "Doi-Koppinen Hopf modules versus entwined modules," New York Journal of Mathematics, vol. 6, pp. 325-329, 2000.

[14] T. Brzeziński, "Deformation of algebra factorisations," Communications in Algebra, vol. 29, no. 2, pp. 737-748, 2001.

[15] S. Dăscălescu and F. Nichita, "Yang-Baxter operators arising from (co)algebra structures," Communications in Algebra, vol. 27, no. 12, pp. 5833-5845, 1999.

[16] F. F. Nichita, Non-Linear Equations, Quantum Groups and Duality Theorems: A Primer on the Yang-Baxter Equation, VDM, 2009.

[17] J. López Peña, F. Panaite, and F. Van Oystaeyen, "General twisting of algebras," Advances in Mathematics, vol. 212, no. 1, pp. 315-337, 2007.

[18] J. C. Baez and A. Lauda, "A prehistory of n-categorical physics," in Deep Beauty: Understanding the Quantum World Through Mathematical Innovation, H. Halvorson, Ed., pp. 13-128, Cambridge University Press, Cambridge, UK, 2011.

[19] J. C. Baez, "Braids and Quantization," http://math.ucr .edu/home/baez/braids.

[20] J. C. Baez, " $R$-commutative geometry and quantization of Poisson algebras," Advances in Mathematics, vol. 95, no. 1, pp. 61-91, 1992.

[21] F. Nichita, "Self-inverse Yang-Baxter operators from (co)algebra structures," Journal of Algebra, vol. 218, no. 2, pp. 738-759, 1999.

[22] P. T. Johnstone, "Adjoint lifting theorems for categories of algebras," The Bulletin of the London Mathematical Society, vol. 7, no. 3, pp. 294-297, 1975.

[23] R. Wisbauer, "Lifting theorems for tensor functors on module categories," Journal of Algebra and its Applications, vol. 10, no. 1, pp. 129-155, 2011.

[24] M. Gerhold, S. Kietzmann, and S. Lachs, "Additive deformations of Braided Hopf algebras," Banach Center Publications, vol. 96, pp. 175-191, 2011.

[25] D. Tambara, "The coendomorphism bialgebra of an algebra," Journal of the Faculty of Science, vol. 37, no. 2, pp. 425-456, 1990. 


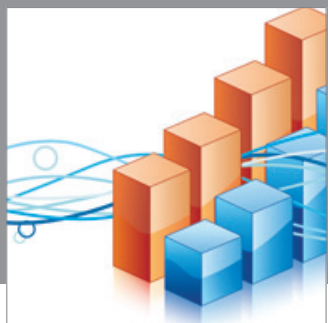

Advances in

Operations Research

mansans

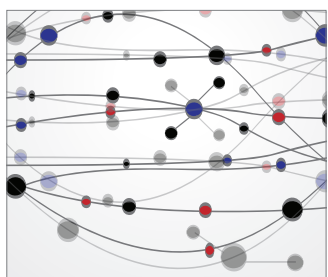

The Scientific World Journal
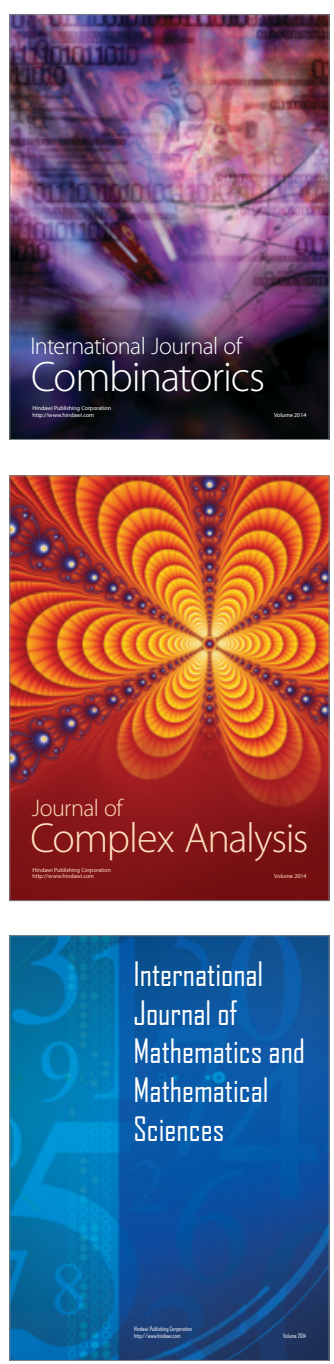
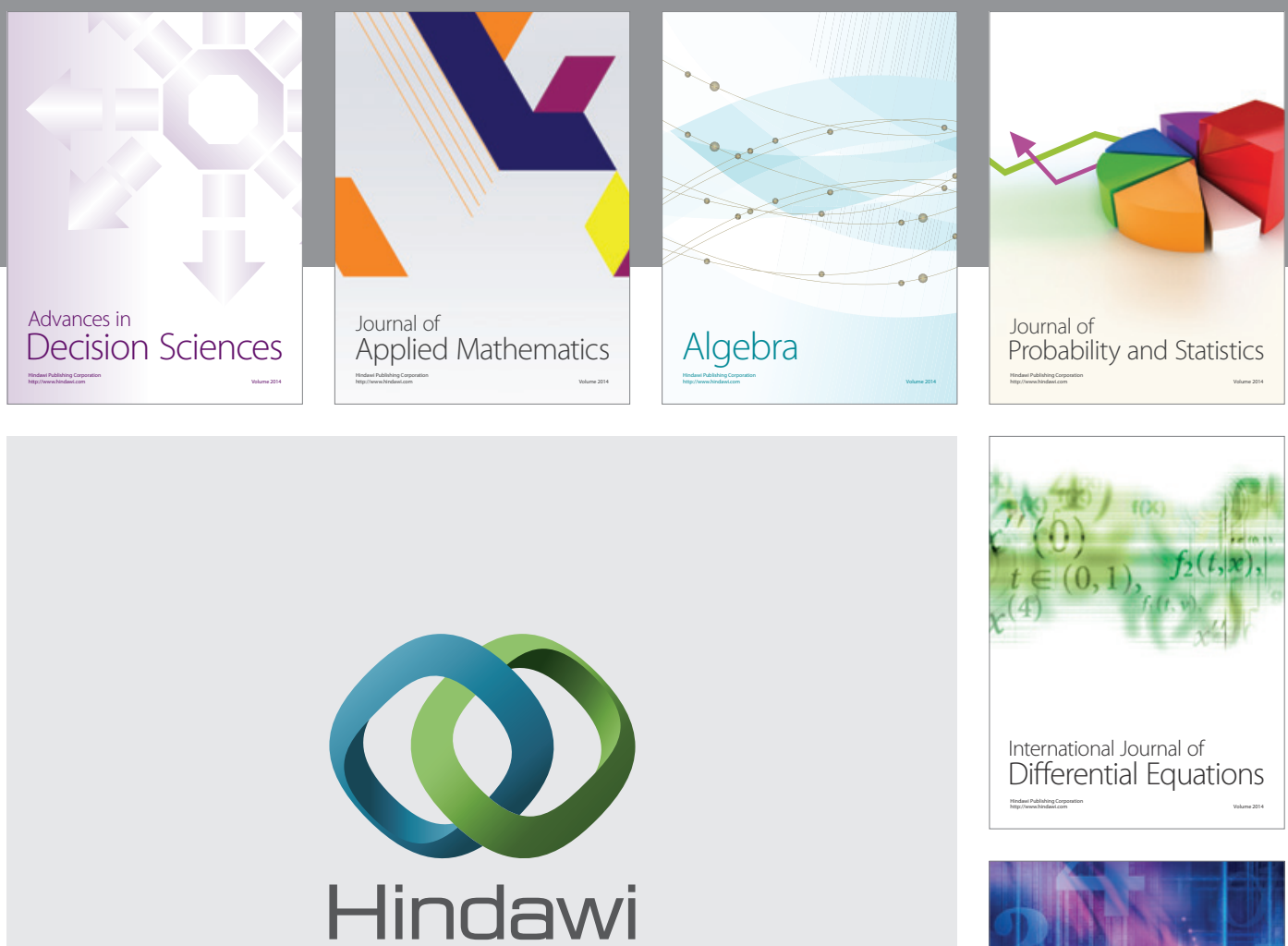

Submit your manuscripts at http://www.hindawi.com
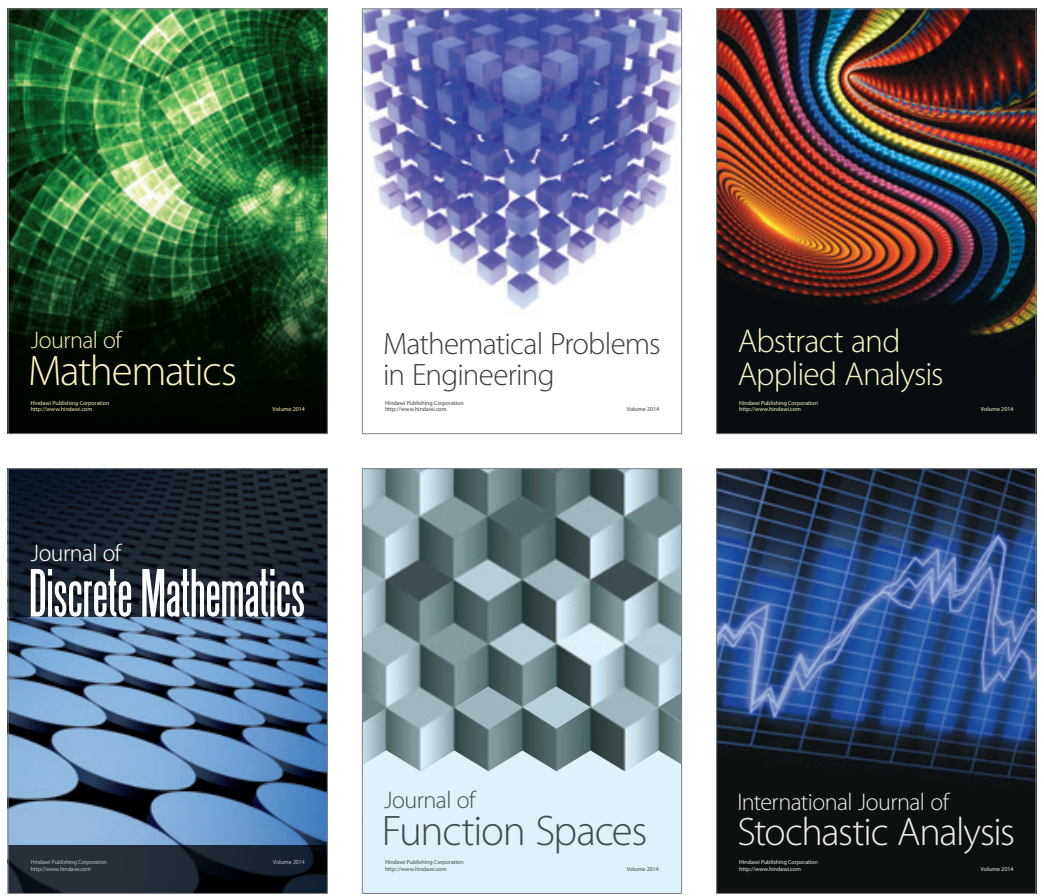

Journal of

Function Spaces

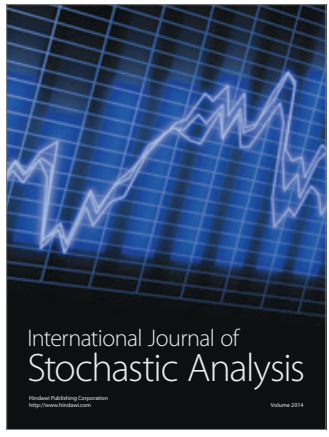

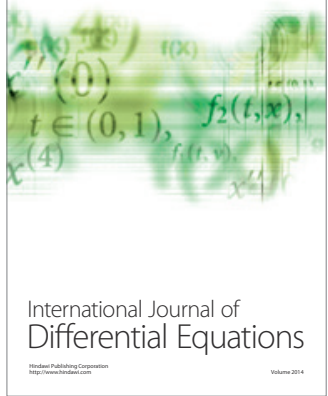
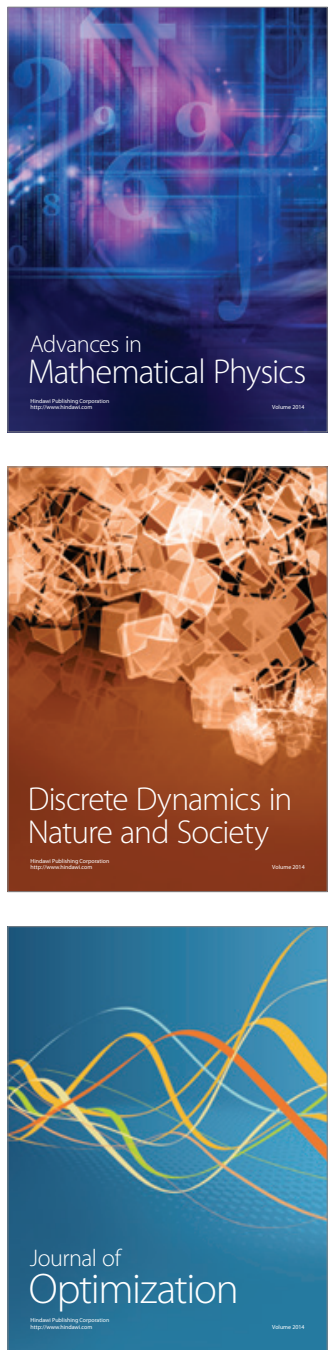